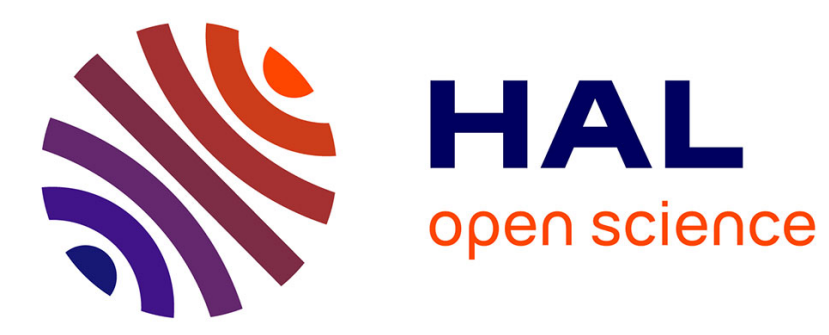

\title{
La divinisation du social
}

Laurent Jaffro

\section{To cite this version:}

Laurent Jaffro. La divinisation du social. M. Fœssel et J.-F. Kervégan. Modernité et sécularisation, CNRS éditions, pp.145-153, 2007. halshs-00174247

\section{HAL Id: halshs-00174247 https://shs.hal.science/halshs-00174247}

Submitted on 6 Jul 2021

HAL is a multi-disciplinary open access archive for the deposit and dissemination of scientific research documents, whether they are published or not. The documents may come from teaching and research institutions in France or abroad, or from public or private research centers.
L'archive ouverte pluridisciplinaire HAL, est destinée au dépôt et à la diffusion de documents scientifiques de niveau recherche, publiés ou non, émanant des établissements d'enseignement et de recherche français ou étrangers, des laboratoires publics ou privés. 
Premier brouillon de «La divinisation du social », in M. Fœssel et J.-F. Kervégan (éd.). Modernité et sécularisation, CNRS éditions, pp. 145-153, 2007.

Laurent JAFFRO, Université Blaise Pascal (Clermont 2) et Institut universitaire de France

La divinisation du social

L'objet de cette contribution est de proposer une notion, celle qui fait son titre, qui, lorsqu'il s'agit de rendre compte de certaines formes particulières de la valorisation du lien social qu'on peut observer depuis le $\mathrm{XVIII}^{\mathrm{e}}$ siècle, pourrait être considérée comme étant préférable à celle de sécularisation. On peut parler d'une «divinisation du social » quand le divin est placé dans la société qui est alors envisagée sous les aspects à la fois de son dynamisme (associatif, collectif, communicationnel) et de son autosubsistance, c'est-à-dire de la croyance selon laquelle le lien social subsiste par lui-même et est doté d'une vertu de régénération. On me dira qu'il suffit de parler simplement de valorisation extrême du lien social. Mais la divinisation est quelque chose de plus que la valorisation, parce qu'on peut très bien valoriser le lien social sans le parer des caractères d'une énergie divine. La divinisation n'est pas n'importe quelle valorisation parce qu'elle se situe, tout comme la sécularisation, à l'intérieur de la question théologico-politique.

La notion de sécularisation correspond à une option théorique qui est pertinente pour décrire des formes relativement déterminées qui vont de la formation des Etats modernes jusqu'à l'instauration de la laïcité ou d'autres modes de pacification des rapports entre les Eglises et l'Etat. Cet énoncé, à savoir que la notion de sécularisation s'applique dans un contexte dans lequel l'existence temporelle est naturellement réalisée à travers des formes étatiques, ne doit pas être confondu avec le fameux théorème de la sécularisation, selon lequel 
les concepts les plus importants de la théorie de l'Etat sont des concepts religieux sécularisés. Il s'agit plutôt du théorème inverse (ou du postulat inverse, puisqu'il n'en sera ici proposé aucune démonstration), selon lequel tous les concepts religieux sécularisés sont relatifs de près ou de loin à la théorie moderne de l'Etat. Les exemples des concepts sécularisés de souveraineté ou de laïcité pourraient constituer une illustration de cette thèse et suggérer que la sécularisation comme «transposition » ne fonctionne que dans des conditions qui, si elles sont générales au sens où on les rencontre abondamment dans l'histoire moderne, restent spécifiques puisqu'elles consistent dans une situation déterminée : celle de la symétrie ou du face-à-face entre deux instances, la respublica et l'ecclesia.

La divinisation du social est une autre option, dont il faut souligner qu'elle peut très bien cohabiter dans l'histoire avec la précédente - l'alternative dont il est question est en effet seulement conceptuelle. La divinisation apparaît quand s'effacent simultanément l'immanence de la société à l'Etat et la transcendance du divin par rapport à la société. A l'inverse, le concept de sécularisation suppose la réalisation intégrale de la société dans l'Etat et l'extériorité du divin, dont le royaume n'est pas de ce monde. En d'autres termes, on peut parler de divinisation du social lorsque le divin est immanent ou coextensif à la socialité et que celle-ci ne se résorbe ou ne s'éduque plus dans une volonté publique proprement politique telle qu'elle s'exprime dans l'Etat. Pourquoi, dans ce contexte, la pertinence de la notion de sécularisation devient-elle très problématique? Parce que la notion de sécularisation s'applique essentiellement et comme par affinité à l'Etat et à ses transformations, ou encore à la société identifiée à l'Etat et non à la société dans sa divergence, et encore moins au lien social quand il est distingué du lien proprement politique.

Mon objet est de construire sommairement la notion de divinisation du social en montrant qu'elle est possible, de la situer par rapport à certaines évolutions du christianisme et aussi par rapport au développement de la société civile au sens contemporain du terme. 
J'insiste sur le fait qu'il ne s'agit pas de substituer globalement la notion de divinisation du social à celle de sécularisation, mais de l'introduire à ses côtés comme un outil plus adéquat quand il s'agit de décrire certains phénomènes qu'on impute ordinairement à la sécularisation. La question de la nécessité du recours à cette notion (en a-t-on vraiment besoin ?) ne sera pas vraiment abordée, ni le détail des cas dans lesquels cette nécessité est sensible.

\section{Une autre spécification de la question théologico-politique}

La sécularisation est une forme particulière de transformation qui se situe dans un ensemble conceptuel beaucoup plus vaste et général qu'on appelle la question théologicopolitique. On s'émerveille parfois que la notion de sécularisation soit profondément religieuse et on y voit toutes sortes de paradoxes stimulants, mais, en réalité, il n'y a pas lieu de s'étonner: si la sécularisation est une espèce de règlement ou de développement de la question théologico-politique, dans la mesure où ce qui se dit du genre se dit aussi de l'espèce, la notion de sécularisation met toujours en rapport le politique et le religieux. Pourquoi n'existerait-il pas d'autres spécifications de la question théologico-politique et, parmi celles-ci, particulièrement, la divinisation du social ?

La notion de divinisation du social comme celle de sécularisation, ai-je dit, prend son sens à l'intérieur de la question théologico-politique. Cette considération suppose alors que l'on donne de la question théologico-politique une interprétation assez large, plus large sans doute que celle que proposait Spinoza. Dans la conception spinoziste qui est exposée et critiquée par Leo Strauss (en fait c'est la conception de toutes les Lumières, pas seulement de Spinoza), les enjeux de la question théologico-politique sont la paix religieuse au sein de l'Etat et aussi et surtout la libertas philosophandi au regard de laquelle la paix religieuse n'est peut-être qu'un moyen. Plusieurs instruments permettent de réaliser cette fin qu'est la 
protection de la liberté de philosopher; le premier de ces instruments est l'interprétation de l'Ecriture ; parmi les autres instruments on trouve, par exemple, l'institution d'une religion civile. On pourrait d'ailleurs voir dans l'anticléricalisme de Spinoza et dans son programme d'émancipation un bon exemple d'une contribution délibérée et militante à la sécularisation.

Mais si l'on veut situer plusieurs formes, pour ce qui nous concerne au moins deux (sécularisation et divinisation), à l'intérieur de l'horizon théologico-politique, il faut bien admettre que celui-ci ne se réduit pas au dispositif somme toute tactique que propose Spinoza dans le Traité théologico-politique. La compréhension la plus générale qu'on puisse donner de la question théologico-politique est la suivante: comment régler les rapports et éventuellement le contentieux entre les deux royaumes, c'est-à-dire entre le divin et le temporel ? Il ne s'agit pas seulement des rapports et du contentieux éventuel entre les représentants ou fonctionnaires de ces deux royaumes, c'est-à-dire entre les prêtres et les magistrats (car cette question-ci correspond à la forme réduite du problème théologicopolitique), mais des rapports et contentieux entre les deux royaumes, bref, entre la société elle-même et la divinité elle-même. Toute compréhension de la question théologico-politique qui viendrait à mettre en doute cette rivalité directe reviendrait à dissoudre la question théologico-politique. Et c'est bien en un sens ce qu'a fait Spinoza dans l'interprétation qu'en donne Leo Strauss, comme le rappelle Daniel Tanguay: Strauss entend rouvrir le débat entre Athènes et Jérusalem que Spinoza avait fermé en lui donnant une solution purement politique $^{1}$

En somme, le concept réduit du théologico-politique, voire la dissolution de ce concept, c'est le concept politique du théologico-politique. La notion de sécularisation est profondément attachée à ce concept politique du théologico-politique. Quant à la divinisation

\footnotetext{
${ }^{1}$ D. Tanguay, Leo Strauss. Une biographie intellectuelle, Paris, Grasset, 2003, p. $221:$ « La solution moderne au problème théologico-politique n'apparaît sérieusement que lorsque les deux rivaux en présence reconnaissent une certaine validité aux prétentions de l'adversaire. De la même manière qu'il a cherché à faire revivre la querelle des Anciens et des Modernes et l'idée du droit naturel, Strauss a tenté aussi de rouvrir le problème théologico-politique. »
} 
du social, elle paraît au contraire relever du concept religieux du théologico-politique, et plus précisément de son concept chrétien. Avec la notion de divinisation, on n'a pas affaire à une simple transposition de notions, mais à une sorte d'hérésie du christianisme - et en ce sens son étude pourrait s'inspirer non certes des thèses de Löwith, mais de la méthode qu'il emploie dans Histoire et Salut.

Pour que la divinisation du social puisse prendre place à l'intérieur de la question théologico-politique, il est nécessaire qu'elle conserve les traits communs de cette question. Par exemple, la relation entre deux royaumes - un royaume qui est de ce monde et un royaume qui n'est pas de ce monde: dans la divinisation du social, cette relation est transformée en une identification des deux royaumes. A la transcendance de la divinité est substituée une immanence prétendument complète qui consiste dans la capacité d'autoconstitution, d'auto-organisation et d'auto-émancipation du social en tant que tel.

Cette option de la divinisation du social n'est pas la même que l'option moderne telle que Strauss la comprend, et que Daniel Tanguay décrit dans ces termes :

Dans la perspective de Strauss, l'option moderne présuppose toujours l'abandon de tout recours à la transcendance et une tentative de fonder la vie politique de manière totalement immanente. De ce point de vue, la modernité est en rupture avec la philosophie ancienne, mais aussi avec les religions révélées, et plus particulièrement avec le christianisme. La colère antithéologique est dirigée contre tous les royaumes imaginaires, autant contre le meilleur régime en paroles des philosophes que contre la Cité de Dieu. ${ }^{2}$

La divinisation du social est aussi une tentation de l'immanence, un « abandon de tout recours à la transcendance », mais sur un plan qui exclut autant le Léviathan que la divinité. La divinisation du social est antipolitique et c'est pour cette raison qu'elle ne saurait être purement antireligieuse et qu'elle n'est certainement plus animée par la «colère

\footnotetext{
${ }^{2}$ Ibid., p. 220.
} 
antithéologique ». Quand on parle d'immanence à propos de l'existence humaine, par opposition à une situation où celle-ci tourne les yeux ailleurs et de préférence vers le haut, il faut aussi se demander à quoi il y a immanence. Bien souvent les théoriciens de la sécularisation opposent immanence et transcendance sans préciser à quel niveau de l'existence temporelle il y a immanence, parce qu'ils sont implicitement convaincus qu'il ne peut s'agir que du niveau politique et institutionnel. La divinisation du social c'est l'immanence du divin à quelque chose qui ne se confond pas avec le Léviathan : le lien social, ce nouveau « royaume imaginaire ».

Il paraît assez clair que Strauss n'a pas vraiment envisagé cette forme. Pourquoi ? Sans doute parce qu'il considérait que l'horizon de l'existence sous son aspect collectif est intégralement politique. Il y a bien dans son œuvre des remarques éparses sur la conception contemporaine d'une socialité dynamique et sympathique ou sur le prêchi-prêcha d'inspiration scoute qui accompagne parfois la démocratie et qui contribue à ce qu'elle soit galvaudée. Il reste cependant convaincu que la forme la plus radicale d'immanence consistait dans une existence purement politique et dans une sorte d'athéisme pratique. Il n'envisage pas véritablement que la tentative contemporaine d' «abandon de tout recours à la transcendance » puisse s'accompagner de l'effacement de l'institution politique au profit de la dynamique sociale ; il envisage encore moins que cette nouvelle configuration puisse être profondément religieuse. Il est vrai que dans le judaïsme de Strauss (et pas seulement de Strauss, bien sûr !) il est impensable qu'on puisse dire que Dieu est parmi nous, sans parler même de son humanisation, et qu'à ce titre la divinisation du social peut être considérée comme un cas mineur de pathologie - tout spécialement un cas de fanatisme chrétien. Même si Strauss a une compréhension profonde de l'histoire politique du christianisme et de l'histoire chrétienne du politique, il demeure, je crois, que sa conception du problème théologico-politique reste toujours attachée à un principe fondamental, selon lequel l'organisation sociale est toujours de 
nature politique et parfois de nature politico-religieuse, mais jamais de nature a-politique ou antipolitique.

C'est sans doute que la notion de divinisation du social ne peut se développer qu'à l'intérieur du christianisme et au terme de certaines évolutions qui sont manifestes depuis le $\mathrm{XVIII}^{\mathrm{e}}$ siècle. La divinisation du social suppose que l'amour de Dieu puisse prendre la forme de l'amour de l'homme, puisqu'elle consiste au fond dans la transformation par laquelle l'amour de l'homme prend le pas sur l'amour de Dieu ; mais il faut préciser que ce qui est aimé dans l'homme, ou à travers lui, n'est pas Dieu, mais l'homme; il faut ajouter que l'homme qui est aimé n'est pas une personne, mais l'humanité même ; et aussi que l'humanité n'est rien d'autre que la société ; et enfin que la société consiste dans la socialité, le dynamisme associatif, le lien en tant que tel.

\section{Au croisement de deux séries de transformations}

Il faut, certes, donner du contenu à cette notion. On peut d'abord faire référence à deux séries convergentes de transformations lentes, qui sont assez sensibles depuis le XVIII ${ }^{\mathrm{e}}$ siècle, et qu'on se borne à indiquer à gros traits :

La première série est celle des transformations du christianisme dans son rapport à la société. Sous cette rubrique, on inclut la socialisation de la religion, la réduction de la religion à une discipline sociale et morale, tant dans les sectes protestantes dont le mode d'organisation est anti-institutionnel que sous la plume des philosophes antireligieux ${ }^{3}$; cette transformation est très sensible chez les quakers et aussi dans le piétisme. Michel de Certeau a montré dans L'Ecriture de l'histoire que l'hétérodoxie religieuse des Lumières s'accompagne d'une orthodoxie morale. Le recul de la dogmatique au profit de la morale est typique des

\footnotetext{
${ }^{3}$ Voir John Toland, La Constitution primitive de l'Eglise chrétienne / The Primitive Constitution of the Christian Church, précédé de L'ecclésiologie de John Toland par L. Jaffro, Paris, Honoré Champion, 2003.
} 
grandes évolutions des Eglises protestantes depuis le XVIII ${ }^{\mathrm{e}}$ siècle; on sait que l'Eglise catholique a connu dans la seconde moitié du $\mathrm{XX}^{\mathrm{e}}$ siècle une évolution similaire. A cette évolution du christianisme s'ajoute, dans l'histoire des théories politiques et des argumentaires de la tolérance, une émancipation de l'ecclesia par rapport à la respublica. L'Eglise est conçue, non plus comme un organe subordonné de la societas par excellence qu'est la république, mais, en dehors de la république, comme une autre societas, infrapolitique, voire antipolitique. La transformation de l'ecclésiologie chrétienne, au premier chef protestante, à l'heure où les Eglises se dépolitisent et se socialisent, converge avec le développement des théories politiques de la tolérance. Au XIX ${ }^{\mathrm{e}}$ siècle, la socialisation du christianisme s'intensifie avec le développement du «christianisme social » qui traverse les confessions ; à quoi il faut joindre aussi la naissance de l'idée d'une religion de l'humanité, sous ses diverses formes, au premier chef dans le positivisme. La divinisation du social peut prendre des formes plus ou moins pacifiques : il ne s'agit pas seulement de l'action sociale chrétienne, il peut s'agir aussi de nouvelles manifestations de l'enthousiasme. On songe à la forme religieuse charismatique, au $\mathrm{XX}^{\mathrm{e}}$ siècle, qui divinise le lien lui-même ou donne une signification presque exclusivement sociale à la communion.

La seconde série est celle des transformations de la société dans son rapport à l'Etat. Cela consiste principalement en l'émergence d'une société civile qui, pour être distincte de la société politique, est d'abord conçue sur le modèle de la société religieuse d'une part, d'autre part des organisations spontanées de l'état de nature. Cette évolution commence dans les théories du contrat (notamment dans la pensée de John Locke), se renforce dans les théories économico-politiques anticontractualistes (David Hume, Adam Ferguson), puis culmine dans les théories de l'association qui foisonnent au $\mathrm{XIX}^{\mathrm{e}}$ siècle. Ici la théorie politique anticipe sur un changement organisationnel de grande ampleur: l'émancipation de la société civile à l'égard des institutions politiques - encore faudrait-il distinguer entre les institutions de l'Etat, 
dont la société civile se détache, et les institutions parapubliques, sur lesquelles elle ne cesse de s'appuyer. Ces changements s'accompagnent de la valorisation d'une affectivité propre à la société civile - l'enthousiasme altruiste, bien thématisé par la philosophie sociale et économique des anticontractualistes écossais - et de modes de coopération spécifiques, qu'on peut résumer avec la notion de solidarité. Bien que ces évolutions proviennent de causalités variées et qu'elles soient peut-être diverses dans leur nature, elles convergent vers un même credo en deux points : la croyance dans le caractère autosubsistant de la société civile ; la croyance selon laquelle l'autosubsistance entraîne un caractère émancipateur.

La divinisation du social est ainsi la rencontre et la consolidation mutuelle de deux séries de transformations, la socialisation du christianisme d'un côté et de l'autre côté la dépolitisation de la société. Naturellement, ces notions de socialisation ou de dépolitisation sont des programmes, voire des slogans ou des mots d'ordre, en tout cas elles sont plus de l'ordre de la valeur que de l'ordre du fait.

\section{Un exemple : Charles Gide et le coopératisme}

A la fin du XIX $\mathrm{X}^{\mathrm{e}}$ siècle en France, le problème théologico-politique est présent non pas seulement à l'intérieur des débats sur la laïcité de l'Etat, sur la liberté de croyance, sur le conflit entre l'appartenance religieuse et l'appartenance politique, sur l'organisation des Eglises, et sur toutes les choses de ce genre qui sont les plus visibles, mais aussi autour d'une question plus discrète : quelle vertu doit accorder à ce qu'on appelle le lien social ? Il faut s'intéresser à la profonde affinité entre certaines théories de l'association et l'ecclésiologie chrétienne. Je ne crois pas qu'on puisse rendre compte de cette affinité dans les termes d'une simple sécularisation de la notion d'église. 
Il s'agit d'un double mouvement. D'une part, le modèle ecclésial s'applique encore à des entités sociales non religieuses. D'autre part, cette application ne laisse pas intacte l'idée de l'Eglise, mais suppose qu'on insiste sur une signification particulière, celle d'une organisation sociale pure. Il n'y a pas de sécularisation au sens d'une transposition de concepts. Mais il y a bien une intériorisation ou une immanentisation du divin. On comprend qu'une telle transformation puisse trouver des conditions très favorables dans certaines conceptions issues de la Réforme, qui tendent à la fois à affirmer que le divin est parmi nous et que l'Eglise à laquelle nous appartenons se réduit à notre association volontaire et temporaire.

Pour ce qui concerne à la fois le développement du christianisme social et d'un socialisme non collectiviste, le cas du coopératisme de Charles Gide et de l'«Ecole de Nîmes » pourrait être exemplaire ${ }^{4}$. Gide lui-même insiste assez sur le lien entre ecclésiologie protestante et coopération :

On peut dire que chaque Eglise protestante constitue comme une sorte de société de consommation spirituelle. Je ne profane pas le mot, car il y a des consommations spirituelles comme il y a des consommations matérielles. La coopération n'a-t-elle pas pour but de pourvoir le mieux possible à tous les besoins de ses membres, si variés soient-ils ? Il peut y

\footnotetext{
${ }^{4}$ Il faut rappeler la situation de l'Eglise réformée au cours du XIX ${ }^{\mathrm{e}}$ siècle. Jusqu'au milieu du siècle, un protestantisme affadi, dit «prélibéral », domine la scène et est caractérisé par le relâchement sur les dogmes, la réduction de l'Evangile à un message moral ou «rationnel », sans que cela donne lieu au développement des œuvres sociales. Dans le même temps, le Réveil venu des évangélistes anglo-saxons et suisses se développe et finit, à partir de 1850, par gagner du terrain à l'intérieur même de l'Eglise réformée. Rétablissant, sinon le calvinisme orthodoxe, du moins certains ses dogmes fondateurs, au premier chef celui de la Chute, les évangéliques favorisent - à Paris souvent en association avec les luthériens -, les œuvres sociales, le « droit au pain » comme le «droit au salut ». Les « libéraux extrêmes », qui joueront un rôle important dans la genèse de la laïcité et des grandes lois républicaines (le meilleur exemple est Ferdinand Buisson), parce qu'ils sont attachés presque exclusivement à la "liberté de conscience », mépriseront la philanthropie évangélique - sur ce sujet, voir Mireille Gueissaz, «Le sentiment de solidarité sociale chez les protestants français au XIX ${ }^{\mathrm{e}}$ siècle », dans Jacques Chevallier et al., La solidarité : un sentiment républicain ?, Paris, PUF, 1992, p. 26-41. A partir des années 70 , les libéraux extrêmes quittent l'Eglise réformée, tandis que les «libéraux modérés » y restent. On classe souvent Charles Gide dans cette dernière catégorie. C'est exact du point de vue « dogmatique ». Mais sa participation active au christianisme social le rapproche aussi du mouvement évangélique ; et la famille Gide était très proche du courant orthodoxe.
} 
avoir et il y a des coopératives pour communications téléphoniques, pour des concerts, pour des représentations théâtrales, et aussi bien pour l'édification mutuelle 5 .

Gide souligne aussi que l'ecclésiologie coopératiste est conforme à l'organisation primitive du christianisme :

On pourrait même pousser plus loin l'assimilation si on voulait remonter aux origines mêmes du christianisme, à ces premiers jours du monde chrétien auxquels le protestantisme revient si volontiers. Les premières communautés chrétiennes ont été des sociétés de consommation, en ce sens qu'elles se réunissaient périodiquement pour des repas en commun, modestes banquets de pauvres gens tels qu'étaient les chrétiens à cette époque. Ces réunions portaient un nom qui est resté dans notre langue, agapes, d'un mot grec qui veut dire amitié, charité, amour. Et c'est dans ces banquets qu'on célébrait la Cène, car la sainte Cène, dans son auguste origine et telle qu'elle fut instituée par Jésus avec ses disciples, n'était pas autre chose qu'un repas dans lequel les convives rompaient ensemble le pain et buvaient à la même coupe ${ }^{6}$.

L'Eglise chrétienne est une sorte de coopérative de consommateurs et la coopérative de consommateurs est une sorte d'église. Cette interprétation communicationnelle ou sociale de la communion est extrêmement importante ; elle est typique de la divinisation du social.

La réduction protestante de l'Eglise à l'association est propice au développement d'une religion de l'humanité ou d'un culte du social en tant que tel. Dans ses leçons de 1924, Gide poursuit l'exposition des motifs pour lesquels «le protestantisme évangélique s'est trouvé orienté vers la coopération ». Il ne s'agit pas seulement des « symboles », mais aussi des «dogmes $»^{7}$. Un premier dogme, « double », est celui de la chute par la faute d'Adam et du salut dans le second Adam, Christ. « De même que la solidarité s'affirme dans la chute, de

\footnotetext{
${ }^{5}$ Le programme coopératiste et le socialisme religieux. Deux leçons du cours sur la coopération au Collège de France, février 1924, Paris, Association pour l'enseignement de la coopération, s. d., p. 16. L'exemple du téléphone prend son sens si on pense à l'extrême rareté de l'équipement à cette époque.

${ }^{6}$ Ibid.

${ }^{7}$ Le programme coopératiste et le socialisme religieux, op. cit., p. 16.
} 
même elle s'affirme dans le salut ». Ainsi, « la devise de la coopération [...] 'chacun pour tous, tous pour chacun' n'est qu'une transposition laïque de la religion chrétienne ellemême ». On remarque que Gide utilise la notion de «transposition laïque » pour décrire ces déplacements, comme s'il adhérait à la thèse de la sécularisation. Mais je crois que ces déplacements vont bien au-delà de la simple transposition.

On pourrait alors montrer de quelle manière une ecclésiologie, donc une christologie, même sous la forme la plus affadie, est présente dans certaines apologies de la solidarité et plus généralement des vertus qu'on attribue à la société civile. La divinisation du lien social qui s'autorise de l'humanisation de Dieu dans le Christ et qui en constitue une interprétation hérétique -, caractéristique de l'ecclésiologie non-conformiste anglo-saxonne et de courants majeurs de la théologie protestante du $\mathrm{XIX}^{\mathrm{e}}$ siècle (contre lesquels s'était élevé un Karl Barth), trouve son prolongement direct dans les conceptions selon lesquelles la vie associative possède par elle-même (c'est-à-dire en raison des valeurs qui sont attachées à son fonctionnement non concurrentiel) la vertu de régénérer toute la vie sociale, politique et économique.

En 1887, décrivant ce qu'il appelait l'« irréligion de l'avenir », Jean-Marie Guyau parlait de l'association comme « ce qui subsistera des religions dans la vie sociale » :

L'idée pratique la plus durable qu'on trouve au fond de l'esprit religieux, comme au fond des tentatives de réforme sociale, est l'idée d'association. A l'origine, nous l'avons vu, la religion est essentiellement sociologique, par sa conception de la «société des dieux et des hommes ». Ce qui subsistera des diverses religions dans l'irréligion future, c'est cette idée que le suprême idéal de l'humanité, et même de la nature, consiste dans l'établissement de rapports sociaux toujours plus étroits entre les êtres ${ }^{8}$.

\footnotetext{
${ }^{8}$ J.-M. Guyau, L'Irréligion de l'avenir, rééd., Paris, Alcan, 1907, p. 339-340.
} 
Guyau insistait sur le rôle du christianisme et de son «Eglise universelle » dans cette transformation. La notion de sécularisation est-elle apte à rendre compte de ce phénomène ? Il existe des formes, dérivées pour l'essentiel du christianisme, qui situent dans le siècle une énergie divine qu'elles confondent avec le dynamisme de la vie sociale et morale. La notion de sécularisation suppose qu'il y a dans l'immanence au siècle quelque chose qui est de l'ordre de la déthéologisation ou de l'antithéologie, comme si la « transposition » des notions religieuses les vidaient au moins partiellement de leurs significations premières. C'est pourquoi la notion de sécularisation doit céder le pas à celle de divinisation du social. Quand la «société des dieux et des hommes » se trouve réalisée dans la «société des hommes », les tenants de la sécularisation donnent à entendre que quelque chose, en chemin, a été « transposé », « sécularisé », bref que les dieux ont été perdus. A rebours, la conception qui a été esquissée ici permet de voir ce que si les dieux sont apparemment absents de cette « société des hommes », c'est qu'elle a été elle-même divinisée. L'irréligion de l'avenir dont Guyau s'était fait le prophète n'est-elle pas, pour nous, la religion présente ? 